\title{
A study on maternal mortality in a tertiary care center in South India
}

\author{
Anupama Suresh $^{1}$, Muralikrishnan Nambiar ${ }^{2}$, Jaice Mary Devasia ${ }^{3 *}$ \\ ${ }^{\mathbf{1}}$ Assosciate Professor, ${ }^{\mathbf{2}, 3}$ Junior Resident, Dept. of Obstetrics and Gynaecology, Kasturba Medical College, Mangalore, Manipal Academy \\ of Higher Education (Deemed University), Karnataka, India \\ *Corresponding Author: Jaice Mary Devasia \\ Email: josemath71@gmail.com
}

Received: $13^{\text {ed }}$ April, 2019

Accepted: $13^{\text {ed }}$ May, 2019

\begin{abstract}
Introduction: Maternal mortality has long been considered as a very good indicator for healthcare quality provided. Pregnancy is considered to be a physiological phase in the lifetime of a woman carrying serious implications on morbidity and mortality. It has been agreed upon that many of the maternal deaths in developing countries can be prevented.

Aim: To study the prevalence of maternal mortality between March 2014-March 2018 in our hospital.

Methods and Materials: It's a retrospective observational study done at a tertiary care centre in southern part of India. Maternal deaths from March 2014 to March 2018 were looked into. Maternal mortality ratio and causes for the maternal deaths were analyzed and compared. Demographic details were collected and data regarding parity, mode of delivery, gestational age at delivery, antenatal checkups, co morbid conditions, causes of death were noted and neonatal outcomes were noted.

Results: Overall live births in the period between March 2014-March 2018 were 18978, of which the number of LSCS were 9590(50.5\%), the number of vaginal deliveries were 9388(49.5\%), the number of maternal deaths were 39(MMR-205/100000 live births).The leading cause was sepsis $48.6 \%$ followed by postpartum hemorrhage (20\%). The most common indirect cause was anemia $(42.85 \%)$.

Conclusion:

Sepsis \& hemorrhage emerged as the greatest killers. Many of the reasons of maternal mortality were found to be preventable. Early identification and stratification of risks with prompt initiation of necessary management measures are necessary to prevent these deaths.
\end{abstract}

Keywords: Maternal mortality, Sepsis, hemorrhage, preeclampsia, direct cause, indirect cause

\section{Introduction}

"Maternal death is defined as the death of a woman while pregnant or within 42 days of termination of pregnancy, irrespective of the duration and site of pregnancy, from any cause related to or aggravated by the pregnancy or its management, but not from accidental or incidental causes". Maternal death is a heavy loss to the family and society. Maternal mortality reflects the health care facilities available to the community. In India maternal mortality statistics has been showing a decreasing trend. ${ }^{2}$ It was at an alarming rate of 677 per one lakh live births in 1980, which fell to 174 per one million live births in $2015,^{3}$ still it is on a high.

In 2015 globally 303,000 women died of various causes related to maternity. ${ }^{4}$ It has been learned that in Northern Europe the risk of a woman dying because of pregnancy and childbirth in her lifetime is about 1 in 30,000 where as in Afghanistan is about 1 in $6 .^{5}$ National rural health mission (NRHM) and Millennium development goal's target is to reduce MMR to less than 100 by 2015 in India. ${ }^{1}$ This was not achieved despite countless measures.

In India the numbers vary drastically from region to region. Socio demographic factors also cast a major influence. A wide variation in the maternal mortality rate has been shown by various studies done in India in the last 15 years ranging from $47 / 100000$ to $625 / 100000 .^{6}$ "What was significant is the data from the state of Karnataka :maternal mortality rate in the state has decreased from 144 in $2011-13$ to 134 in 2017-18."3
Mortality occurring due to complications related to pregnancy, labor and the puerperium, which can be due to commission and omission of interventions, injudicious treatment or may be due to combination of these factors is termed as the direct causes of obstetric death. Deaths as a result of previously existing condition or a disease, that first occurred during pregnancy which are not direct obstetric causes. ${ }^{7}$ Indirect causes of maternal mortality can be worsened by physiological changes of pregnancy. Maternal deaths are most commonly caused by complications associated with pregnancy or delivery or a combination of both. ${ }^{8}$ These complications include hemorrhage, unsafe abortions, infections postpartum, hypertensive disorders during pregnancy, obstructed labour etc which accounts for more than $75-80 \%$ of direct maternal deaths. ${ }^{9}$ The risk multiplies when more than one factor is involved.

Pregnancy is not a disease but a physiological state; hence pregnancy related mortality is almost always preventable. ${ }^{10}$ But this demands a great amount of effort and caution from us. We need to have more of institutional data to pin point the various lacunae that led to the increase in maternal mortality over the years in this southern state of India.

The purpose of orchestration of this study is to determine the magnitude of maternal mortality and the various factors contributing to it in our tertiary care centre.

\section{Aim}

To study the prevalence of maternal mortality during March 2014-March 2018 in a tertiary care centre. 


\section{Materials and Methodology \\ Study setting}

Government Lady Goschen Hospital Mangalore and KMC Hospital, Attavar, Mangalore.

It is a retrospective observational study done in a tertiary care centre in Dakshina Kannada district of Karnataka, India. Maternal deaths from March 2014 to March 2018 were studied. Maternal mortality ratio and causes for the maternal deaths were analysed and compared. Demographic details were noted including age, parity, mode of delivery, gestational age at delivery, antenatal checkups, pregnancy related complications, long standing co morbid conditions, causes of death and neonatal outcomes were noted.

\section{Results}

Overall live births in the period between March 2014 to March 2018 were 18978, of which the number of LSCS were 9590 and remaining were vaginal deliveries, the number of maternal deaths were 39(MMR-205/100000 live births). Highest mortality was recorded in the months of October, (6) November (5) and December (8).

Maximum number of mortality was in the age group of 26-30 years (48.7\%). Fifteen $(38.4 \%)$ of the 39 were primigravida and remaining $(61.5 \%)$ were multigravidas.

Among the 39 maternal deaths, $46 \%$ had vaginal delivery and $46 \%$ had lower segment caesarean section and 3 patients were not delivered $(7.7 \%)$. 46\% (18) delivered after term gestation. $79.4 \%$ had regular ANCs. $35.9 \%$ had co morbid conditions of which anemia constituted the majority i.e., six mothers $(42.85 \%)$. The number of mothers who had regular antenatal care and delivered in our hospital were sixteen patients $(41 \%)$, antenatal care elsewhere and delivered in our set up were 13(33.3\%), antenatal care and delivery elsewhere and referred to our centre were 6 $(15.38 \%)$ and those with no antenatal care were $4(10.2 \%)$.

Direct causes that resulted in maternal deaths were thirty five $(89.7 \%)$. Sepsis proved to be the biggest contributor to maternal mortality in our centre, resulting in seventeen $(48.6 \%)$ maternal deaths followed by postpartum hemorrhage and amniotic fluid embolism which caused seven $(20 \%)$ and four $(11.4 \%)$ maternal deaths respectively. Venous thromboembolism and ante partum hemorrhage resulted in one death $(2.85 \%)$ each. Eclampsia resulted in two deaths $(5.7 \%)$. Indirect causes leading to maternal death were Guillain-Barre syndrome, peripartum cardiomyopathy, pulmonary artery hypertension and carcinoma breast with extensive metastasis each resulting in one maternal death each. Of the mothers who died fourteen (35.9\%) had chronic co morbid conditions of which anemia was identified to be the most prevalent with six $(42.85 \%)$ mothers featuring anemia followed by diabetes (14.3\%) and chronic hypertension $(14.3 \%)$

Of the 39 maternal deaths that occurred, 25(64\%) had surviving live births without any complications or needing management in a neonatal intensive care unit, 13(33.3\%) were intrauterine deaths/ still born and one neonatal death $(2.56 \%)$.
Table 1: Observed co morbid conditions in the mothers who died (in percentage).

\begin{tabular}{|l|c|c|}
\hline \multicolumn{1}{|c|}{ Comorbidity } & N & Percentage \\
\hline Chronic hypertension & 2 & 14 \\
\hline Gestational diabetes & 2 & 14 \\
\hline Anemia & 6 & 43 \\
\hline Depression & 1 & 7 \\
\hline Malignancy & 1 & 7 \\
\hline Cardiac disease & 1 & 7 \\
\hline thyrotoxicosis & 1 & 7 \\
\hline
\end{tabular}

Table 2: Causes resulting in maternal mortality

\begin{tabular}{|c|c|c|}
\hline Cause & N & Percentage \\
\hline Direct & 35 & 90 \\
\hline Indirect & 4 & 10 \\
\hline
\end{tabular}

Table 3: Direct causes resulting in maternal mortality

\begin{tabular}{|l|c|c|}
\hline \multicolumn{1}{|c|}{ Cause } & N & Percentage \\
\hline Amniotic fluid embolism & 4 & 11 \\
\hline Venous thromboembolism & 1 & 3 \\
\hline sepsis & 17 & 49 \\
\hline Eclampsia & 2 & 6 \\
\hline Antepartum haemorrhage & 1 & 3 \\
\hline Postpartum haemorrhage & 7 & 20 \\
\hline metastasis & 1 & 3 \\
\hline Cardiac cause & 2 & 6 \\
\hline
\end{tabular}

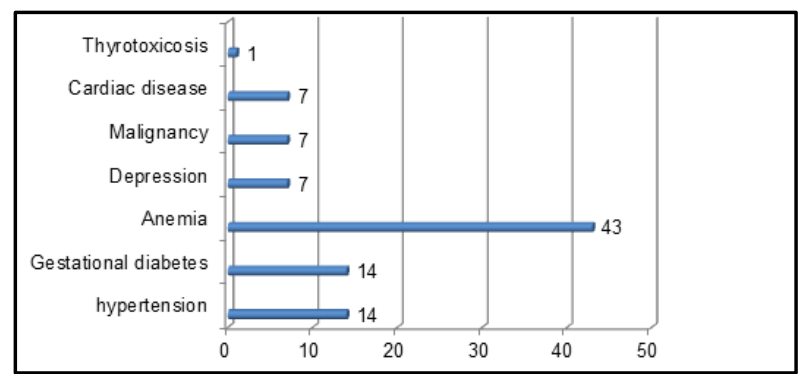

Fig. 1: Co-morbidities in pregnancy".

\section{Discussion}

In this study majority of the deaths belonged to 26-30 years age group, which can be due to the social situation seen in our community, where most of the pregnancies happen in this age group. At the same time it is noteworthy that teenage pregnancies and advanced maternal age did not pose a major threat to maternal health at least in this study setting in the prescribed time period. This is due to the decreased rate of teenage pregnancies in this part of Karnataka. It is in contradiction to the findings in many other studies. ${ }^{11}$ In the National representative survey between 2001-2003 by the registrar general of India of the 1096 maternal deaths (which accounted for $11.9 \%$ of all deaths of women in the age group between 15-49 years), half the deaths were in the age group of 20-29 years with a median age of 26 years. ${ }^{12}$ This is similar to the observation made in this study.

The caesarean section rate in our hospital during the study period was 50.5\%(9590). This is about five fold 
higher than the national average of $10 \% .^{7}$ This could be mainly associated with the fact that our hospital is a tertiary care centre with most of the cases being referred here for various complications. The lower segment caesarean section rate among the mothers who died was $18(46 \%)$ and remaining had vaginal deliveries $18(46 \%)$ and 3 were not delivered (7.7\%). None of the deaths resulted as a direct consequence of the surgical complications. None of the studies have compared the mode of delivery conducted and its relation with maternal mortality.

$46 \%$ of the deaths occurred at term gestation, this is comparable to other studies where most of the complications leading to maternal mortality arose at term gestation. A study by Pratima devi et al had shown that majority of death had occurred at term $(57.5 \%)^{16}$ which is comparable to this study.

The number of mothers who had regular antenatal care and delivered in our hospital were sixteen $(41 \%)$, ante natal care elsewhere and delivered in our set up were 13(33.3\%), antenatal care and delivery elsewhere and referred to our centre were six $(15.38 \%)$ and those with no antenatal care were $4(10.2 \%)$. It was noted by Montgomery et al that mothers who had more than one antenatal visit was about $58.3 \%$ which is comparable in this scenario ${ }^{7}$. This undermines the importance of regular and meticulous antenatal care with deep insight into any associated high risk factors which plays an important role in prevention and early identification of complications leading to maternal deaths. ${ }^{13}$

In our study direct causes that resulted in maternal deaths were thirty five $(89.7 \%)$. Sepsis proved to be the biggest contributor to maternal mortality in our centre, resulting in seventeen $(48.6 \%)$ maternal deaths followed by postpartum hemorrhage and amniotic fluid embolism which caused seven $(20 \%)$ and four $(11.4 \%)$ maternal deaths respectively. Venous thromboembolism, metastasis and ante partum hemorrhage resulted in one death $(2.85 \%)$ each. Eclampsia resulted in two deaths $(5.7 \%)$. This finding agrees with most of the other studies as well as the general consensus in this regard. One such study observed that more than one fourth of the maternal deaths were due to obstetric hemorrhage $(\mathrm{n}=296)$ with most occurring in the intrapartum period $(\mathrm{n}=258)$ followed by sepsis $(\mathrm{n}=184) .^{7} \mathrm{~A}$ study done by Abilasha et al showed that $67.7 \%$ of maternal deaths are due to direct causes and common direct causes encountered where hemorrhage $(33.8 \%)$,eclampsia $(10.3 \%)$ and sepsis $(8.2 \%) .{ }^{17}$ This data is not corresponding to what we have seen in this particular study.

Indirect causes leading to maternal death in our study were Guillain Barre Syndrome, peripartum cardiomyopathy, pulmonary artery hypertension and carcinoma breast with extensive metastasis each resulting in one maternal death each. These conditions may vary from place to place and time to time. These conditions need multi-disciplinary involvement for adequate management and do not always limit itself to obstetric management alone. ${ }^{14}$ Pratima et al have found higher incidence of cardiovascular causes as major indirect factor which could not be elicited in this study. ${ }^{16}$

We found that fourteen (35.9\%) of the mothers who died had chronic co morbid conditions among which anemia was identified to be the most prevalent with six (42.85\%) mothers featuring anemia followed by diabetes $(14.3 \%)$ and chronic hypertension (14.3\%). It has to be noted that despite the multiple programs by the central and state Government anemia still has high prevalence and poses a great threat to maternal health. ${ }^{15}$ we have found an higher prevalence of anemia in mothers who died when compared to studies done by Pratima et al $(15 \%)^{16}$ and Abilasha et al $(14.7 \%) .{ }^{17}$ Anemia is one condition which can be the causative factor for almost all obstetric complications leading to maternal death. As previously discussed, regular antenatal care can alleviate the burden of anemia in mothers who are at high risk developing anemia.

\section{Conclusion}

1. Maternal mortality ratio in our hospital is 205 which is quite high. This is probably because our hospital is a tertiary care centre where many cases are referred in view of complications.

2. Sepsis is the major cause and more emphasis should be put on undertaking all the necessary measures for asepsis.

3. Timely referral to tertiary care centers should be encouraged in primary health care facility.

4. Regular maternal death reviews need to be conducted to look into the causes and take necessary steps to prevent them in future.

\section{Conflict of Interest: None.}

\section{Reference}

1. Bhanot BM. Parks textbook of preventive and social medicine Indicators of MCH care. 20th ed. 2009. 479 p.

2. WHO, UNICEF, UNFPA WB. Trends in maternal mortality: 1990 to 2010. Geneva: World Health Organization. 2012.

3. R 2011-13. RGI (SRS 2011-13)/state wise information / Karnataka. 2015. p. nrhm.gov.in/nrhm-in-state/state wise information /.

4. Alkema L, Chou D, Hogan D, Zhang S, Moller A-B, Gemmill A et al. Global, regional, and national levels and trends in maternal mortality between 1990 and 2015, with scenariobased projections to 2030: a systematic analysis by the UN Maternal Mortality Estimation Inter-Agency Group. Lancet 2016;387(10017):462-74.

5. Ronsmans C, Graham WJ. Maternal mortality: who, when, where, and why. Lancet 2006;368(9542):1189-1200.

6. Asha J, Govind RP. Maternal mortality - changing trends 2007;57(5):398-400.

7. Montgomery AL, Ram U, Kumar R, Jha P, Death M. Maternal Mortality in India : Causes and Healthcare Service Use Based on a Nationally Representative Survey PLoS One 2014;9(1).

8. Rogo KO, Oucho J, Mwalali P, Jamison DT, Feachem RG, Makgoba MW et al. Disease and Mortality in Sub-Saharan Africa. Int Bank Reconstr Dev / World Bank 2006. 2006;(16).

9. Berhan Y, Berhan A. Causes of Maternal Mortality in Ethiopia: A Significant Decline in Abortion Related Death. Ethiop J Health Sci 2014;24(0):15-28.

10. Jadhav CA, Prabhakar G, Shinde MA, Tirankar VR. Original 
article Maternal Mortality: Five Year Experience in Tertiary Care Centre. Indian J Basic Appl Med Res 2013;(7):702-6.

11. Gibbs, C. M., Wendt, A., Peters, S., \& Hogue CJ. The Impact of Early Age at First Childbirth on Maternal and Infant Health. Paediatr Perinat Epidemiol 2012;26(1):259-84.

12. Office of Registrar General I. Annual Health Survey (AHS) in 8 EAG States and Assam - Release of District Level Factsheet: 2010-11 Evidence based planning requires. 2012.

13. Roy, M. P., Mohan, U., Singh, S. K., Singh, V. K., \&Srivastava AK. Determinants of Utilization of Antenatal Care Services in Rural Lucknow. India. J Fam Med Prim Care 2013;2(1):55-9.

14. Knight M, Nair M, Tuffnell D, Kenyon S, Shakespeare J, Brocklehurst P KJ (Eds. on behalf of, MBRRACE-UK. Saving Lives, Improving Mothers' Care - Surveillance of maternal deaths in the UK 2012-14 and lessons learned to inform maternity care from the UK and Ireland Confidential Enquiries into Maternal Deaths and Morbidity 2009-14. Oxford
NatlPerinat Epidemiol Unit, Univ Oxford. 2016;

15. Mangla M, Singla D. Prevalence of anaemia among pregnant women in rural India : a longitudinal observational study. Int $J$ Reprod Contracept Obstet Gynecol 2016;5(10):3500-5.

16. Pratima D, Manglem S, Randhoni D. Maternal mortality and its causes in atertiary centre. J Obstet Gynaecol India 2012:62(2);168-71

17. Nair A, Doibale MK, Gujrathi VV, Inamdar IF, Shingare AD, Rajput PS. Study of maternal mortality in a tertiary center hospital in a district of Maharashtra. Int J Med Sci Oublic Health 2016;5:1851-54.

How to cite this article: Suresh A, Nambiar M, Devasia $\mathrm{JM}$, A study on maternal mortality in a tertiary care center in South India. Indian $J$ Obstet Gynecol Res 2019;6(2):173-176. 